\title{
A KINETIC MODEL FOR VEHICULAR TRAFFIC DERIVED FROM A STOCHASTIC MICROSCOPIC MODEL
}

\author{
by $\mathrm{R}$. Wegener and A. Klar \\ Fachbereich Mathematik \\ Universität Kaiserslautern \\ 67663 Kaiserslautern \\ Germany
}

\begin{abstract}
A way to derive consistently kinetic models for vehicular traffic from microscopic follow the leader models is presented. The obtained class of kinetic equations is investigated. Explicit examples for kinetic models are developed with a particular emphasis on obtaining models that give realistic results. For space homogeneous traffic flow situations numerical examples are given including stationary distributions and fundamental diagrams.
\end{abstract}

\section{Introduction}

Modelling and simulation play an increasing role in the optimization of traffic flow. Traditionally there have been three types of approaches towards the problem. The first and most basic one concerns microscopic or follow the 
leader models, modelling the actual response of single cars to their predecessor, see, e.g., [1, 2, 3, 4]. Macroscopic models based on fluid dynamic equations have been proposed by a large number of authors, see, e.g., [5, 6, 7, 8, 9, 10]. However some of these models have been subject to a considerable controversy, concerning their validity and applicability to traffic flow. Kinetic or Boltzmann-like models may present an intermediate step between the above two types of models. On the one side they can be derived from microscopic considerations. On the other side at least some macroscopic models can be derived from kinetic traffic models as has been shown, e.g. in $[11,12,13]$.

Kinetic models contain more variables than macroscopic models and less variables than microscopic ones. Therefore, one expects that computation times for the simulation of kinetic models range between those for microscopic and those for macroscopic models. However, we mention here a certain type of microscopic models, the cellular automaton models. They need simulation times comparable to those of macroscopic models, see for example $[14,15]$.

The first kinetic traffic models were published by Prigogine and Andrews [16, 17], who introduced a Boltzmann-like term to account for the slowing down interactions (see Prigogine and Herman [11] for a review). However, they and most of their successors, see, e.g., [18, 13, 12], treated the acceleration of the cars by means of a heuristic relaxation term. Only recently Nelson [19] succeeded in obtaining reasonable kinetic equations by using a kinetic description also for acceleration. The derivation of his equation is strictly based on a microscopic model in contrast to the Prigogine work. He also proved that his model fulfills the criterion of having a one parameter family of local equilibrium distributions depending only on the local density of cars. However, as he himself states, his model is a caricature of traffic flow and should only be seen as a first step in obtaining a kinetic equation that is also suitable for real applications.

In the present work we aim at deriving a kinetic equation that is strictly based on a microscopic model without going back to phenomenological relaxation terms as in the Prigogine model. The model should however be capable of giving reasonable results and describing real traffic flow patterns.

The paper is organized in the following way: Section 2 describes the microscopic models that we use as the basic description of traffic flow. Section 3 shows how kinetic models are derived from this type of microscopic models. In section 4 we describe an explicit kinetic model derived from a very simple microscopic model. However, as can be seen from the numerical simulations in section 5, the kinetic model describes already all important features of homogeneous traffic flow. Finally, in section 5 we describe a numerical scheme to solve 
the kinetic equation and present some numerical results for the space homogeneous equation. The numerical investigation of the spatially inhomogeneous case of real traffic flow situations will be considered in future work.

\section{Microscopic Theories}

In this section we describe the stochastic microscopic model underlying our kinetic model.

Usually in microscopic models the response of a car to the behaviour of its leading vehicle is considered, see [3] and references therein. The drivers are assumed to change their velocity only in response to the leading car. This assumption is certainly not justified for traffic flow at very low density. However, once the number of cars is not too low it seems to be reasonable. Moreover the situation of traffic flow with very low density does not seem to be of very much interest in traffic flow applications.

If a vehicle is faster than the leading car and the headway to the leading car is becoming smaller than a certain threshold, its driver is slowing down or passing the leading vehicle. If the vehicle is slower than the leading car and the headway to the leading car is becoming larger than another threshold, the car is accelerating. Actually several different thresholds for slowing down and acceleration procedures are known. One observes, for example, thresholds based on a certain desired distance or on a minimal acceptable distance between a driver and his leading vehicle, see $[1,4]$. The threshold will in general depend on the velocity of the car and on the velocity of the leading vehicle. The motion of the cars is normally described by a second order ordinary differential equation, e.g.,

$$
\frac{d^{2} x_{k}}{d t^{2}}=a_{k}\left(x_{k}, x_{k+1}, \frac{d x_{k}}{d t}, t, \ldots\right),
$$

where an instantaneous change of the acceleration is assumed, if the vehicle $k$ is crossing one of the different thresholds.

In our microscopic model we allow stochastically distributed decisions. The drivers may react in several different ways, e.g., slowing down to certain velocities below the velocity of the leading vehicle. Moreover, to obtain a kinetic equation, velocity changes due to interaction in the microscopic model are as-

sumed to happen instantaneously. This is certainly a simplification, but more or less justified on the considered time scales. 
Surprisingly even the simplest reasonable examples of such models basing on just two thresholds - a slowing down and an acceleration line - give already reasonable results, once they are translated into a kinetic equation. This is demonstrated in section 5 . In particular, we are able to obtain plausible results for the velocity distribution functions and the so called fundamental diagram of homogeneous traffic flow in dependence of vehicle density.

\section{The microscopic model}

Consider a car 1 at place $x_{1}$ with the velocity $v_{1}$ and its leading car 2 at $x_{2}$ with velocity $v_{2} . v_{1}$ and $v_{2}$ are assumed to be in $[0, w]$, where $w$ denotes the maximal velocity.

Let $N$ be the number of thresholds under consideration. If car 1 is crossing a threshold $i \in\{1, \ldots N\}$, i.e., if the headway $h=x_{2}-x_{1}$ is becoming larger or smaller than a certain threshold $h=H_{i}\left(v_{1}, v_{2}\right)$, then car 1 is changing its velocity to the new velocity $v . v_{1}$ and $v_{2}$ are, for each threshold $i$, out of a certain range $\Omega_{i}$ of values associated with the threshold $H_{i}$. The new velocity

is taken on instantaneously in accordance with a certain distribution function

$$
\sigma_{i}\left(v ; v_{1}, v_{2}\right)
$$

$\left(v_{1}, v_{2}\right) \in \Omega_{i} . \sigma_{i}$ may also depend on $x$ and $t$, however, for simplicity of notation we do not write this explicitely. Since $\sigma_{i}$ is a density function it has to fulfill

$$
\int_{0}^{w} \sigma_{i}\left(v ; v_{1}, v_{2}\right) d v=1
$$

A slowing down maneuver could be given, e.g., by a function $H_{i}\left(v_{1}, v_{2}\right)=\epsilon\left(v_{2}\right)$, where $\epsilon$ represents the minimal acceptable distance to a leading vehicle with speed $v_{2}$. Since a car is slowing down only if its velocity is larger than that of his leading vehicle, $\Omega_{i}$ is in this case given by $\left\{\left(v_{1}, v_{2}\right) ; v_{1}>v_{2}\right\}$. In particular, one can recover the Prigogine slowing-down term by setting $H_{i}=0, \sigma_{i}$ equal to a delta distribution $\delta\left(v-v_{2}\right)$, and $\Omega_{i}=\left\{\left(v_{1}, v_{2}\right) ; v_{1}>v_{2}\right\}$.

\section{Remark}

In the microscopic model of Nelson [19] the drivers adapt their velocities not only due to the crossing of a threshold but also due to unexpected events, e.g., instanteneous changes of the velocity of the predecessor. In contrast, a vehicle changes its velocity in our model only if a threshold is crossed. 


\section{Derivation of Kinetic Theories}

In this section we will derive from the microscopic model in section 2 a kinetic equation. The derivation is done by arguments similiar to the derivation of the Boltzmann equation in gas dynamics, see [20] or [21], as follows:

Let $f(x, v, t)$ denote the phase-space density, i.e., the distribution function of the number of vehicles at place $x$ and time $t$ with velocity $v$ multiplied by the density. Writing down the equation for the change of the total number of vehicles leads as usual to the kinetic equation:

$$
f_{t}+v f_{x}=\left(\frac{\delta f}{\delta t}\right)_{g}-\left(\frac{\delta f}{\delta t}\right)_{l}
$$

where $t \in[0, \infty), v \in[0, w]$, and $\left(\frac{\delta f}{\delta t}\right)_{g}$ and $\left(\frac{\delta f}{\delta t}\right)_{l}$, are gain and loss terms due to discontinuous velocity changes, respectively. We can write them as

$$
\left(\frac{\delta f}{\delta t}\right)_{g}=\sum_{i=1}^{N}\left(\frac{\delta f}{\delta t}\right)_{g}^{i},\left(\frac{\delta f}{\delta t}\right)_{l}=\sum_{i=1}^{N}\left(\frac{\delta f}{\delta t}\right)_{l}^{i},
$$

where $\left(\frac{\delta f}{\delta t}\right)_{g}^{i}$ and $\left(\frac{\delta f}{\delta t}\right)_{l}^{i}$ are describing the gain and loss terms that are due to the i-th threshold. We mention that in section 4 we will restrict to the case of two thresholds corresponding to acceleration and slowing-down interactions. The gain and loss terms are given by

$$
\begin{aligned}
& \left(\frac{\delta f}{\delta t}\right)_{g}=\sum_{i=1}^{N} \int_{\Omega_{i}}\left|v_{1}-v_{2}\right| \sigma_{i}\left(v ; v_{1}, v_{2}\right) f_{2}\left(H_{i}\left(v_{1}, v_{2}\right), v_{2}, x, v_{1}, t\right) d v_{1} d v_{2} \\
& \left(\frac{\delta f}{\delta t}\right)_{l}=\sum_{i=1}^{N} \int_{\left(v, v_{2}\right) \in \Omega_{i}}\left|v-v_{2}\right| f_{2}\left(H_{i}\left(v_{1}, v_{2}\right), v_{2}, x, v, t\right) d v_{2} .
\end{aligned}
$$

$f_{2}$ denotes the pair distribution function

$$
f_{2}\left(h, v_{2}, x, v_{1}, t\right)=g\left(h, v_{2} ; x, v_{1}, t\right) f\left(x, v_{1}, t\right),
$$

where $g\left(h, v_{2} ; x, v_{1}, t\right)$ denotes the distribution of the leading vehicles with headway $h$ and velocity $v_{2}$ for a vehicle with velocity $v_{1}$, space $x$ at time $t$.

To obtain from these equations a closed equation for $f$ we have to express $g$ by $f$. This can be done along the lines of Nelson [19], who introduced 
a correlation model using the following basic assumption which he termed 'generalized vehicular chaos assumption':

Let $m\left(h ; x, v_{1}, t\right)$ be the probability density for the leading vehicles at headway $h$, i.e.

$$
m\left(h ; x, v_{1}, t\right)=\int_{0}^{w} g\left(h, v_{2} ; x, v_{1}, t\right) d v_{2} .
$$

Let the density of vehicles at place $x$ and time $t$ be given by $\rho(x, t)$, i.e.

$$
\rho(x, t)=\int_{0}^{w} f(x, v, t) d v .
$$

Then, the generalized vehicular chaos assumption states that

$$
\frac{g\left(h, v_{2} ; x, v_{1}, t\right)}{m\left(h ; x, v_{1}, t\right)}=\frac{f\left(x, v_{2}, t\right)}{\rho(x, t)} .
$$

In addition we assume that $m$ does not depend on $v_{1}$. On $x$ and $t$ it is assumed to depend only via $\rho(x, t)$, i.e. $m$ can be written as

$$
m\left(h ; x, v_{1}, t\right)=\rho(x, t) k(h, \rho(x, t))
$$

Then $g$ has the form

$$
g\left(h, v_{2} ; x, v_{1}, t\right)=f\left(x, v_{2}, t\right) k(h, \rho)
$$

Without using an explicit form for $k$ we obtain thus the equation

$$
f_{t}+v f_{x}=Q(f)=\left(\frac{\delta f}{\delta t}\right)_{g}-\left(\frac{\delta f}{\delta t}\right)_{l}
$$

with

$$
\begin{aligned}
&\left(\frac{\delta f}{\delta t}\right)_{g}= \sum_{i=1}^{N} \int_{\left(v_{1}, v_{2}\right) \in \Omega_{i}}\left|v_{1}-v_{2}\right| \sigma_{i}\left(v ; v_{1}, v_{2}\right) f\left(x, v_{1}, t\right) f\left(x, v_{2}, t\right) \\
& k\left(H_{i}\left(v_{1}, v_{2}\right), \rho\right) d v_{1} d v_{2} \\
&\left(\frac{\delta f}{\delta t}\right)_{l}=f(x, v, t) \sum_{i=1}^{N} \int_{\left(v, v_{2}\right) \in \Omega_{i}}\left|v-v_{2}\right| f\left(x, v_{2}, t\right) k\left(H_{i}\left(v, v_{2}\right), \rho\right) d v_{2}
\end{aligned}
$$

Nelson used the following explicit form for $k$ :

$$
k(h, \rho)=e^{-\rho\left(h-\epsilon_{0}\right)},
$$


where $\epsilon_{0}$ is the desired headway at speed 0 . This is for numerical calculations somewhat to restrictive. Looking at numerical simulations it seems to be more appropriate to assume a function $k(h, \rho)$ that is not so much concentrated around the minimal headway.

We remark that, similar to Enskogs theory of a dense gas, one actually would have to introduce a relation like

$$
g\left(h, v_{2} ; x, v_{1}, t\right)=f\left(x+h, v_{2}, t\right) \tilde{k}(x, h, t) .
$$

to take into account that the leading vehicle is at the point $x+h$. This is important, if inhomogeneous traffic flow is considered. However, in the present paper we restrict to an investigation of the homogeneous equation

$$
f_{t}=Q(f)
$$

where $f=f(v, t)$ with some initial condition

$$
f(v, 0)=f_{0}(v) .
$$

We do not consider inhomogeneous traffic, i.e.the influence of the convection term $v f_{x}$. This will be done in a forthcoming publication, see also [22].

Considering the homogeneous equation we remark first of all that an integration over $v$ of the interaction term $Q(f)(v)$ gives 0 , since $\int \sigma_{i}\left(v ; v_{1}, v_{2}\right) d v=$ $1, i=1, \ldots, N$. This means that the number of vehicles is not changed by interactions. A basic result about existence and uniqueness of the homogeneous equation can be easily obtained under slight additional assumptions. This can be done analogously to the classical proof in kinetic theory, see, e.g., Cercignani [20]. This proof shows as well that the density is a conserved quantity during the evolution of the distribution function.

An interesting question is now to investigate, whether the obtained homogeneous equation has a one parameter family of stationary distributions depending only on the density. This leads to unique relation between the mean velocity - computed from the above stationary distribution at a certain density - and the density. This is true for certain classes of kinetic equation, see [20]. For the above general model, where $\sigma_{i}$ is not specified, it is certainly not true and the stationary distributions may depend on the particular initial conditions. However, for a simplified model, as in section 5, numerical results indicate that actually the stationary distribution depends only on the density.

We mention that for real life traffic flow the independence of the stationary distribution from the inital distribution seems not to be supported by empirical data. In particular the scattering of data in empirically obtained fundamental diagrams seems to indicate a dependence on the initial values. 


\section{An Explicit Kinetic Model}

In this section we present an explicit example for the kinetic models derived in section 3 . The aim is here to obtain a model that is as simple as possible but reproduces already the essential features of homogeneous traffic flow. We assume only two threshold, one for slowing down and one for acceleration interactions.

\section{Slowing Down Interactions}

In the simplest case the slowing down threshold is given by a headway

$$
h=H_{1}\left(v_{1}, v_{2}\right)=\epsilon_{1},
$$

where $\epsilon_{1}$ is a positive constant. For slowing down $\Omega_{1}$ is given by

$$
\Omega_{1}=\left\{\left(v_{1}, v_{2}\right) \in[0, w]^{2} ; v_{1}>v_{2}\right\}
$$

The slowing down term is modeled by a Prigogine-like term. However we do not restrict to slowing down to the actual speed of the leading vehicle as in the Prigogine work, but to a range of speeds smaller than this one. We allow slowing down of car 1 to a velocity $v \in\left[\beta v_{2}, v_{2}\right] . \beta$ is some positive constant smaller than 1 . In this range a uniform distribution of velocities is assumed due to the lack of a more precise knowledge.

If the following car is faster than the leading one, we have - alternatively to slowing down - also to take into account the possibility of passing with a certain probability $P$. We assume the velocity of the passing car to remain the same as before. This gives

$$
\sigma_{1}\left(v ; v_{1}, v_{2}\right)=P \delta\left(v_{1}-v\right)+(1-P) \frac{1}{v_{2}(1-\beta)} \chi_{\left[\beta v_{2}, v_{2}\right]}(v)
$$

where $\delta(v)$ is the delta function at $v$ and $\chi_{[a, b]}$ is the characteristic function of the interval $[a, b]$.

The probability of passing $P$ depends on $\rho$ like

$$
P=1-\frac{\rho}{\rho_{\max }}
$$

where $\rho_{\max }$ stands for the maximal vehicular density. This relation was already assumed by Prigogine and Herman, see [11]. 


\section{Acceleration Interactions:}

The acceleration threshold is also assumed to be given by a constant

$$
h=H_{2}\left(v_{1}, v_{2}\right)=\epsilon_{2},
$$

where $\epsilon_{2}$ is positive. For acceleration $\Omega_{2}$ is given by

$$
\Omega_{2}=\left\{\left(v_{1}, v_{2}\right) \in[0, w]^{2} ; v_{1}<v_{2}\right\} .
$$

We assume that car 1 accelerates from its actual speed $v_{1}$ to a certain range of speeds between $v_{1}$ and $v_{1}+\alpha\left(w-v_{1}\right)$. Moreover, we assume again due to the lack of a more precise knowledge that the velocity after acceleration is uniformly distributed in the range $\left[v_{1}, v_{1}+\alpha\left(w-v_{1}\right)\right]$. This leads to the distribution function

$$
\sigma_{2}\left(v ; v_{1}, v_{2}\right)=\frac{1}{\alpha\left(w-v_{1}\right)} \chi_{\left[v_{1}, v_{1}+\alpha\left(w-v_{1}\right)\right]}(v) .
$$

Since for dense traffic the possibility of acceleration is more restricted than for traffic flow with a low density, $\alpha$ is supposed to depend on the density in the following way:

$$
\alpha=\alpha_{0}\left(1-\frac{\rho}{\rho_{\max }}\right),
$$

where $\alpha_{0} \leq 1$ is some positive constant.

Moreover we assume that $k\left(\epsilon_{1}, \rho\right)$ and $k\left(\epsilon_{2}, \rho\right)$ are equal, i.e.

$$
k\left(\epsilon_{1}, \rho\right)=k\left(\epsilon_{2}, \rho\right)=\kappa(\rho) .
$$

This leads for the homogeneous equation to the same influence of the correlation terms on acceleration and slowing down. In this case the correlation terms are only influencing the speed of relaxation to the stationary distribution function in the homogeneous equation, see section 5 .

\section{Remark 1}

$v_{1} \rightarrow v_{1}+\frac{\alpha}{2}\left(w-v_{1}\right)$ is the average increase of velocity for each individual car during the time interval between two acceleration interactions. This corresponds to an acceleration

$$
a=\nu \frac{\alpha}{2}\left(w-v_{1}\right),
$$

where $\nu$ is the acceleration-interaction frequency. With $\frac{1}{T}=\nu \frac{\alpha}{2}$ this can be written as

$$
a=\frac{\left(w-v_{1}\right)}{T},
$$

which corresponds to the Paveri-Fontana acceleration term, see [13]. 


\section{Remark 2}

Note, that in contrast to the Prigogine theory, we did not introduce a distribution function of desired velocities but used a uniform desired velocity $w$. Therefore the number of parameters compared to the Prigogine-equation is considerably decreased. Introduction of a desired speed distribution is particularly reasonable for very low densities of cars, where the distribution of the velocities does not depend on the interactions between the vehicles, but essentially on the drivers wishes. However, for higher densities the drivers wishes should not play a decisive role in contrast to the drivers response to their leading vehicles. We remark finally that an introduction of a desired speed distribution would avoid the singularity in the equation at $v=w$.

Moreover, it would be possible to include in the model a further variable for modelling the individual desired speeds of the drivers similarly to the treatment by Paveri-Fontana [13].

\section{Numerical Results for the Homogeneous Case}

In the following the model in section 4 is numerically investigated. The equation under consideration is

$$
f_{t}=Q(f)
$$

with

$$
\begin{aligned}
\frac{1}{\kappa} Q(f)(v) & =\int_{0}^{w} \int_{0}^{w}\left|v_{1}-v_{2}\right| \sigma\left(v ; v_{1}, v_{2}\right) f\left(v_{1}, t\right) f\left(v_{2}, t\right) d v_{1} d v_{2} \\
& -f(v, t) \int_{0}^{w}\left|v-v_{2}\right| f\left(v_{2}, t\right) d v_{2}
\end{aligned}
$$

where

$$
\sigma\left(v ; v_{1}, v_{2}\right)= \begin{cases}\sigma_{1}\left(v ; v_{1}, v_{2}\right), & \text { if } v_{1}>v_{2} \\ \sigma_{2}\left(v ; v_{1}, v_{2}\right), & \text { if } v_{1}<v_{2}\end{cases}
$$

$\sigma_{1}$ and $\sigma_{2}$ depend on the density $\rho=\int_{0}^{w} f(v, t) d v$ as defined in the last section. We remark again that the density is a conserved quantity of the equation. $\kappa=\kappa(\rho)$ was defined in the last section.

We mention here that for the simulation of the homogeneous equation a further specification of $\kappa$ is not necessary, since different $\kappa$ result only in a change of the time scale in the homogeneous equation. 
We treat the kinetic equation by a discretization scheme, that is described in the following:

A simple standard discretization of the equation in velocity-space needs a large number of discretization points in order to describe correctly the influence of the singularities appearing at $v=0$ and $v=w$. Therefore we divide the velocity space into a certain number of cells and calculate the transition rates between the cells given by the above kinetic equation. Introducing gridpoints $v_{i}=\frac{i}{N}, i=0, \cdots, N-1$ ( $w$ is set equal to 1$)$ and the values $f_{i}=f\left(v_{i}\right)$ the discretized equation is given by

$$
\partial_{t} f_{i}=\frac{1}{N^{3}} \sum_{j, k=0}^{N-1} \bar{\sigma}_{i, j, k}|j-k| f_{j} f_{k}-\frac{1}{N^{2}} f_{i} \sum_{k=0}^{N-1}|j-k| f_{k},
$$

with

$$
\bar{\sigma}_{i, j, k}=N^{3} \int_{(i, j, k) / N}^{(i+1, j+1, k+1) / N} \sigma\left(v ; v_{1}, v_{2}\right) d v d v_{1} d v_{2},
$$

where $\bar{\sigma}_{i, j, k}$ is evaluated analytically. The time derivative is treated in a standard way. This means we implement a discrete velocity approximation of the kinetic equation using the discrete transition rates $\bar{\sigma}_{i, j, k}$ which are averaged values for each cell. Therefore the scheme needs a much lower number of cells than a standard discretization scheme due to the analytic treatment of the singularities. Thus, considerably less computing time is necessary to obtain the same results. Moreover, and this is most important, the density

$$
\rho=\frac{1}{N} \sum_{i=0}^{N-1} f_{i}
$$

is automatically preserved in the scheme, since

$$
\frac{1}{N} \sum_{i=0}^{N-1} \bar{\sigma}_{i, j, k}=1
$$

The stationary distribution $f_{\text {stat }}(v)$ of the above homogeneous kinetic equation turns out to depend only on the density of the initial distribution, but not on the initial velocity distribution.

In all calculations we used a number of cells $N=50$. In the first picture we show a normalized version of the stationary distribution $F(v)=f_{\text {stat }}(v) / \rho$ for different values of $\rho$. The units are taken such that $w$ and $\rho_{\max }$ are equal to 1 . $\alpha_{0}$ and $\beta$ have been chosen equal to 0.3 . 


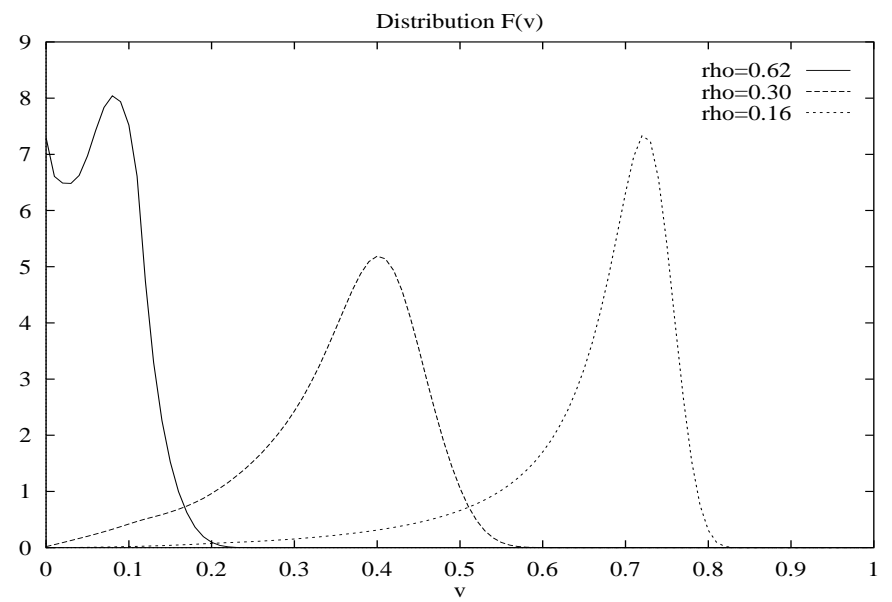

Figure 1: Stationary distributions for $\rho=0.62,0.3,0.16$

In the next two pictures the fundamental diagrams are shown. We plotted the mean velocity

$$
V(\rho)=\int_{0}^{w} v F(v) d v
$$

and the flux

$$
q(\rho)=\rho V(\rho)
$$

versus the density for the whole range of densities.

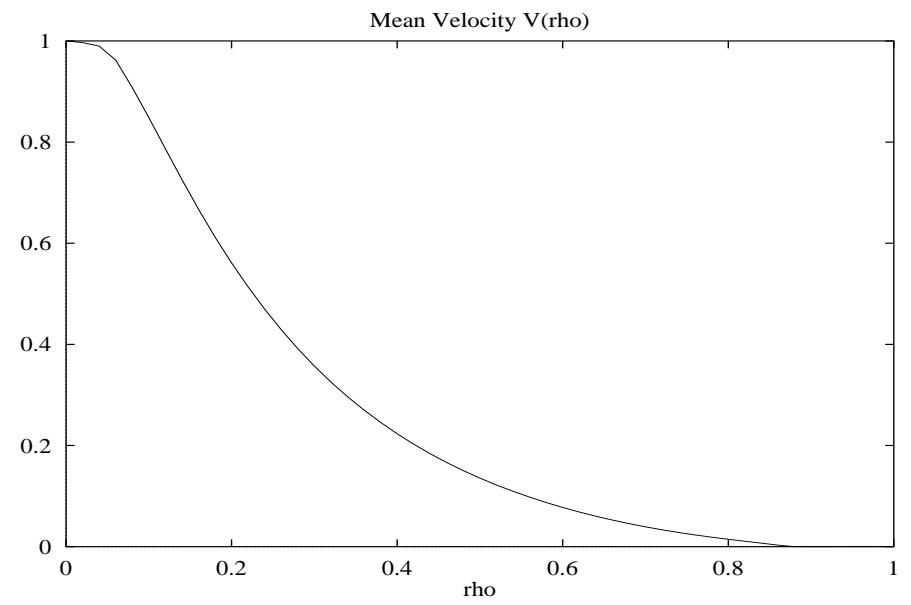

Figure 2: Mean velocity $\mathrm{V}$ in dependence of density $\rho$ for stationary and spatially homogeneous traffic 


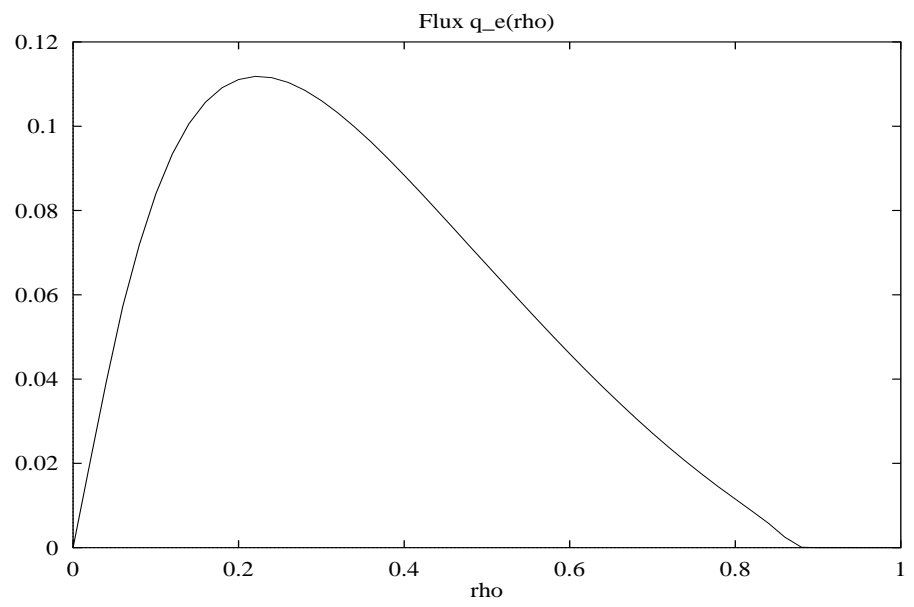

Figure 3: Fundamental diagram, i.e. flux $q$ in dependence of density $\rho$ for stationary and spatially homogeneous traffic

We remark that the fact that the flux is 0 for $\rho$ values bigger than 0.9 is due to the discretization. Using more cells one would obtain a smooth decay to 0 . By adjusting the parameters $\alpha_{0}$ and $\beta$ a whole range of fundamental diagrams may be obtained. The parameters can be chosen such that the fundamental diagrams fit very well to different traffic flow situtations. Several different fits to the actually measured fundamental diagrams are reported, see, e.g. [7].

\section{Acknowledgements}

We are grateful to H. Kühne, P. Nelson and H. Neunzert for interesting discussions and informations. Moreover we wish to thank an unknown referee for helpful hints improving the the paper considerably.

\section{References}

[1] R. Wiedemann. Simulation des Straßenverkehrsflusses. Schriftenreihe des Instituts für Verkehrswesen der Universität Karlruhe, 1974.

[2] D.L.Gerlough and M.J. Huber. Traffic Flow Theory. Transportation Research Board Special Report 1965, Washington D.C., 1975.

[3] D.C. Gazis, R.Herman, and R. Rothery. Nonlinear follow-the-leader models for traffic flow. Opns. Res., 9:545, 1961. 
[4] N. Anstett. Entwicklung eines ereignisorientierten Fahrzeug-FolgeModells zur mikroskopischen Verkehrssimulation. Diplomarbeit Universität Stuttgart/Daimler Benz AG, 1992.

[5] D. Helbing. An improved fluid dynamic model for vehicular traffic. to appear in Physical Review E.

[6] B.S. Kerner and P.Konhäuser. Physical Review E, 50:54, 1994.

[7] R.D. Kühne and Rödiger. Proceedings of the 1991 Winter Simulation Conference, Phoenix, Arizona, 1991.

[8] R.D. Kühne. Verkehrsablauf auf Fernstraßen. Phys. Bl., 47/3:201, 1991.

[9] H.J. Payne. Transportation Research Record, 722:68, 1979.

[10] G.B. Witham. Linear and Nonlinear Waves. Wiley, New York, 1974.

[11] I. Prigogine and R. Herman. Kinetic Theory of Vehicular Traffic. American Elsevier Publishing Co., New York, 1st edition, 1971.

[12] W.F. Phillips. Kinetic Model for Traffic Flow. National Technical Information Service, Springfield, Virginia, 1977.

[13] S.L. Paveri-Fontana. On Boltzmann like treatments for traffic flow. Transportation Research, 9:225, 1979.

[14] K. Nagel and A. Schleicher. Parallel Computing, 20:125, 1994.

[15] M. Schreckenberg, A. Schadschneider, K. Nagel, and N. Ito. Physical Review E, 51:2939, 1995.

[16] I. Prigogine and F.C. Andrews. A Boltzmann like approach for traffic flow. Oper. Res., 8:789, 1960.

[17] I. Prigogine. A Boltzmann like approach to the statistical theory of traffic flow. In R. Herman, editor, Theory of traffic flow, page 158, 1961.

[18] M. Lampis. Transportation Science, 12:16, 1978.

[19] P. Nelson. A kinetic model of vehicular traffic and its associated bimodal equilibrium solutions. to appear in Transp. Theory and Stat. Phys., 1994.

[20] C. Cercignani. The Boltzmann Equation and its Applications. Springer, New York, 1st edition, 1988. 
[21] J. Keitzer. Statistical Thermodynamics of Nonequilibrium Processes. Springer, New York, 1987.

[22] A. Klar, R.D. Kühne, and R. Wegener. Mathematical models for vehicular traffic. to appear in Surv. Math. Ind., 1996. 


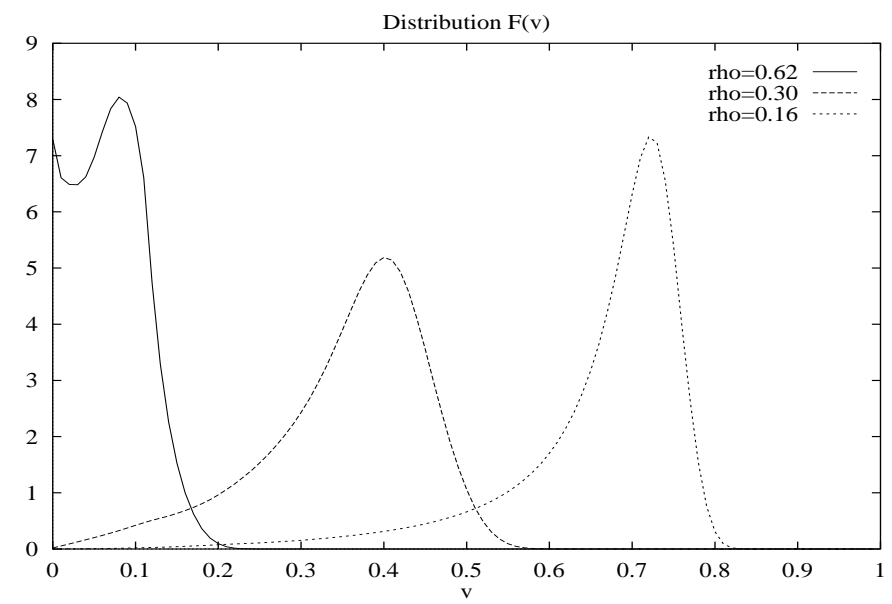




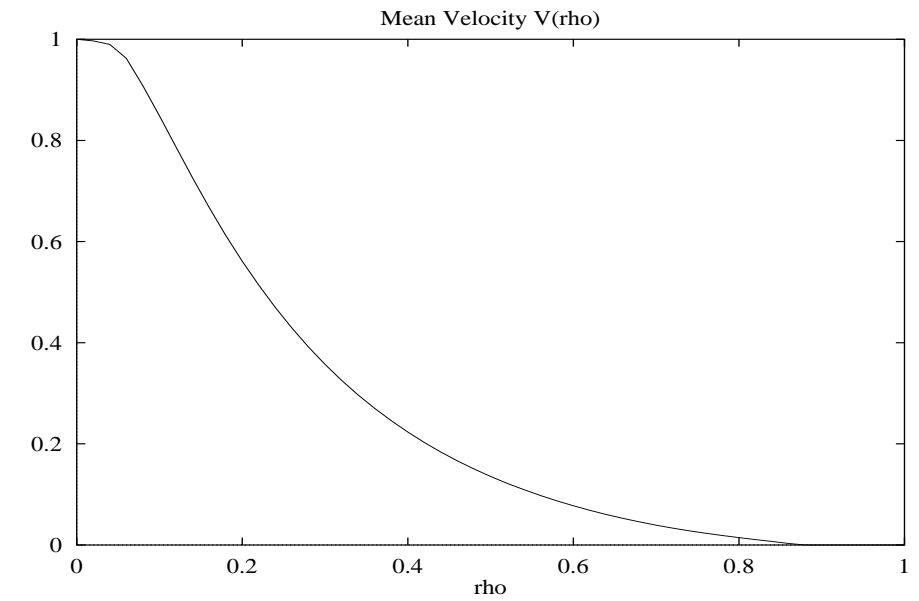




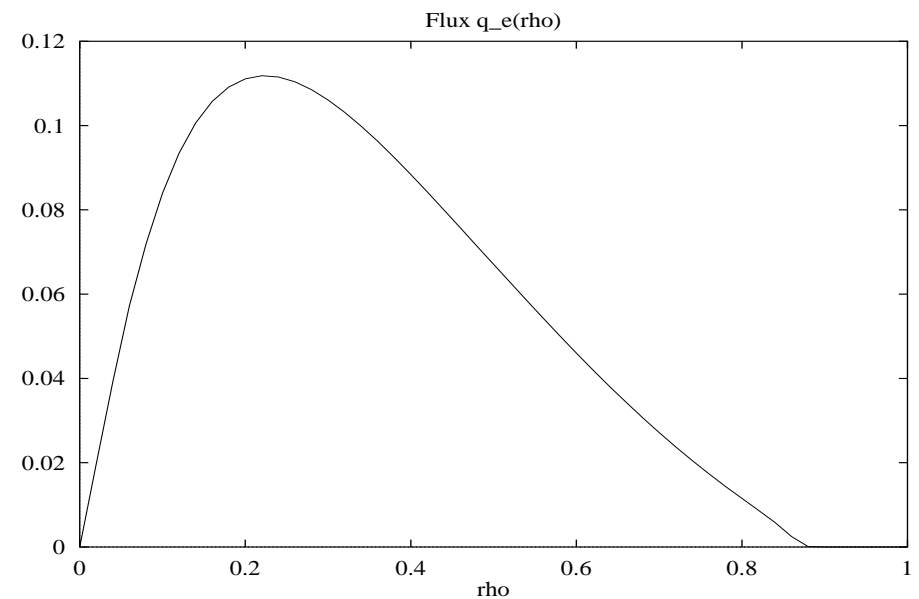

